\title{
Pediatric tracheostomy: complications and role of home care in a developing country
}

\author{
Sadaf Zia - Muhammad Arshad - Zafar Nazir • \\ Sohail Awan
}

Accepted: 24 September 2009/Published online: 16 October 2009

(C) Springer-Verlag 2009

\begin{abstract}
Introduction Tracheotomy in its earlier days was most commonly performed for acute airway infection in children. Its indications are now changing; it is now most commonly performed for congenital malformations (McMurray and Prescott in Practical pediatric otolaryngology. W.B. Saunders Company, Philadelphia, pp 575$592,1996)$. This shift in indication has increased the rate of survival of such patients, and therefore the number of children going home after tracheostomy has also increased. Objective This study was conducted (1) to observe the pattern of indication and complications for tracheostomy, in our part of the world, (2) the rate at which tracheostomy can help wean patients off the ventilator, and (3) the feasibility of sending these children home with tracheostomy. Materials and methods A retrospective study was done on 127 patients. The indications, final outcome and the complications encountered in and outside the hospital were studied through review of charts.

Results Based on the main indications, patients were grouped into: prolonged ventilation group (PV) 61\%, followed by mechanical obstruction group (MO) $22 \%$, and the
\end{abstract}

S. Zia $(\bowtie) \cdot$ S. Awan

Department of Otolaryngology Head and Neck Surgery,

Aga Khan University Hospital, Karachi, Pakistan

e-mail: Sadafzia979@yahoo.com

S. Awan

e-mail: sohail.awan@aku.edu

M. Arshad · Z. Nazir

Department of Pediatric Surgery,

Aga Khan University Hospital, Karachi, Pakistan

e-mail: arshad.muhammad@aku.edu

Z. Nazir

e-mail: zafar.nazir@aku.edu last being adjunct to surgery group (AS) 17\%. The in-hospital complication rate was $30 \%$ and that at home was $18.11 \%$. The most common complications included upper respiratory tract infections, and blockage or displacement of tubes. The late complication rate was $4 \%$. Hundred (78.8\%) patients on the ventilator could be successfully weaned off, with a $p$ value of $0.001 ; 81$ were sent home with the tracheostomy tube (TT). Forty of these were successfully decannulated and the overall decannulation rate was $48.8 \%$. Conclusion A large number of tracheostomies have been performed in the PV group to reduce the intensive care unit (ICU) stay and to prevent nosocomial infections. The need arises from the high cost of prolonged stay in an ICU setup, which is a cause of major economic burden, and lack of financial assistance for these patients worsens the scenario. Home care of the tracheostomy tubes remains a good option for patients requiring long periods of time to overcome their primary pathology.

Keywords Pediatric tracheostomy - Complications · Home care

\section{Introduction}

Tracheostomy is one of the earliest surgical procedures performed; Hippocrates made the first description of making a hole in the airway to relieve upper airway obstruction [1]. Alexander the Great saved the life of one of his soldiers from suffocation by making an opening in the trachea using the tip of his sword [2]. Until 1825, only 28 successful tracheostomy tubes (TT) have been verified in literature [2]. It was popularized during the 1950 s when children with diphtheria were saved by performing tracheostomy [1] Surgical management of airway has now become an 
elective procedure and is performed routinely for mechanical obstruction (MO) internationally [3]. But, in our country it is performed for pulmonary toilet $[2,4,5]$, to facilitate weaning of children from mechanical ventilation.

Pediatric tracheostomy involves major responsibility on the part of the surgeon as well as parents, especially if it involves labor-intensive home care, which includes deprivation of sleep and social isolation of parents [1]. Home care allows a more normal social adjustment of child and is less expensive than extended hospitalization [6]. It allows parents and their children to have a more normal life away from hospital environment, permitting some children to join schools [7]. Along with the benefits, there are also some areas of concern, such as hours of sleep a parent can have with such a child and the number of caretakers involved in home management of the child with TT.

In developing countries such as Pakistan, we felt that there was a need to study the problems faced by pediatric patients and their parents during their care at the hospital and at home. We needed to assess the management in our own social and economic background. Our objective was to study the pattern of indication and complications in our part of the world and to look at the feasibility of sending these children home with TT. Also, the effect of tracheostomy in weaning patients off the ventilator was studied.

\section{Materials and methods}

Study design

This was a retrospective review of medical records of children younger than 14 years of age, who underwent tracheostomy in a single tertiary teaching hospital from 1988 to 2003. The variables of age at the time of tracheostomy, indication for performing the tracheostomy, underlying medical conditions, duration of ventilation before and after tracheostomy, hospital stay, complications in hospital and at home and any tracheostomy-related death was retrieved from the records. Patient records were retrieved with the help of ICD-9 coding 31.1, 31.2 and 31.74 .

The statistical tests of frequency, mean, median and standard deviation were applied. The Chi-square test was applied for comparing data and $p$ value was calculated. A $p$ value of $<0.05$ was considered to be significant.

Patients going home with TT were given instructions and training regarding care and changing of TT in the hospital. They were provided with a spare TT, suction catheters and a manual foot paddle-operated suction machine, as electrical outages, for hours at a stretch, are a common problem in our country. Patients were discharged only when the caregiver and physician were comfortable that the degree of training required for the management of the tube was adequate.

\section{Results}

A total of 127 patients with a mean age of 43 months were included in the study. The male to female ratio was $2: 1$. The patients were divided into three main groups according to the indication for tracheostomy as (1) mechanical obstruction (MO) group $(n=30,22 \%)$ (2) adjunct to surgery (AS) group $(n=20,17 \%)$ (3) prolonged ventilation (PV) group $(n=77,61 \%)$.

For the MO group $(n=30)$, indications are given in Fig. 1. The age of these patients ranged from 4 days to 2 years with a mean of 7.2 months. This group had the longest hospital stay ranging from 9 to 649 days, with a mean of 363 days. The most common complication in the hospital was pneumonia (PN) followed by bilateral pneumothorax (PT). Four cases of tracheo-cutaneous fistula (TCF) were encountered in this group following decannulation (Table 2). There was one death in the hospital secondary to tube obstruction leading to PT (Table 1). Out of 29 patients sent home with TT, 17 (56\%) were decannulated subsequently. Two deaths at home were related to TT: the first was related to TT obstruction, and in the other the cause of death was uncertain, as patient was brought dead to the hospital (Table 2). Two other deaths at home were secondary to other causes.

Twenty patients (17\%) in the AS to surgery group had an age range of 4 months to 14 years with a mean age of 8 years. The tracheostomy was kept for a mean duration of 23 days, which was the shortest duration of TT being kept in place. The indications are given in Fig. 1. Foreign body aspiration was an unusual indication noted in this group: one tracheostomy was done as an emergency measure to secure airway after failed attempts at intubation and bronchoscopy. The patient had aspirated plastic pebbles, which were impacted in the right main bronchus. There was marked edema and bleeding at the glottis, which had caused failed intubation. One patient had a whistle and another had aspirated a ragged iron pebble, which were causing trauma at the subglottis; therefore, tracheostomy was done to retrieve the foreign bodies and protect the subglottis from more trauma. Fourteen $(70 \%)$ patients of this group were decannulated in hospital and discharged (Table 1). The complications are given in Table 1 . This group did not have any home-related mortality, though there was one death in the hospital following accidental dislodgement. The patients discharged with TT in this group did not have any follow-up, as probably they went back to their home towns, or a change of address or phone numbers did not allow any further contact with these patients. 


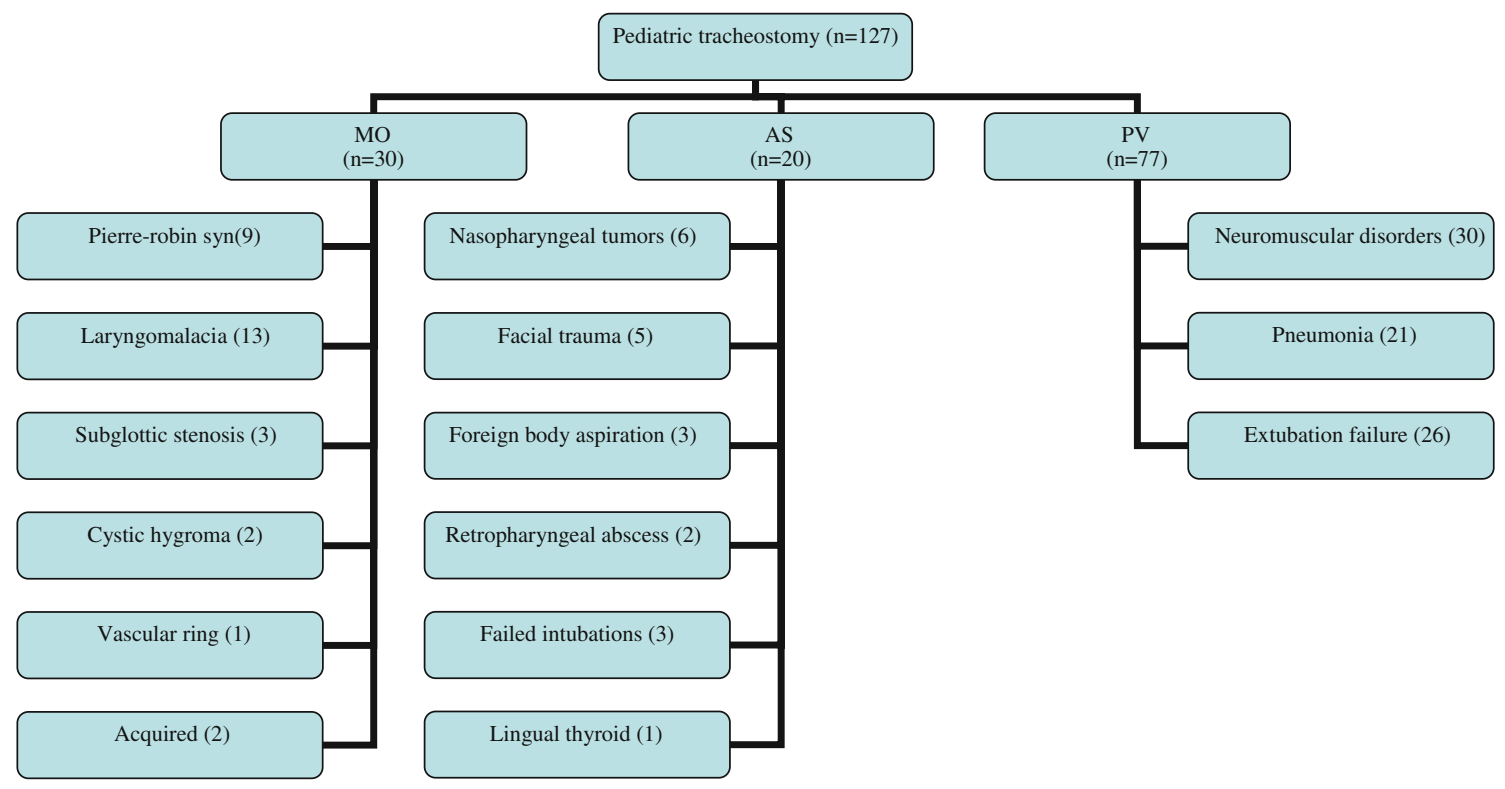

Fig. 1 Indications of pediatric tracheostomy. $M O$ mechanical obstruction, $A S$ adjunct to surgery, $P V$ prolonged ventilation group

Table 1 Complications, decannulation rate and mortality rate in hospital $(n=127)$

\begin{tabular}{|c|c|c|c|c|c|c|c|c|c|}
\hline & \multirow[t]{2}{*}{$\mathrm{AD}$} & \multirow[t]{2}{*}{$\mathrm{CO}$} & \multirow[t]{2}{*}{ PT } & \multirow[t]{2}{*}{ PM } & \multirow[t]{2}{*}{$\mathrm{PN}$} & \multirow[t]{2}{*}{ DR } & \multicolumn{3}{|c|}{ In-hospital mortality } \\
\hline & & & & & & & RTT & URTT & $\%$ \\
\hline MO group $n=30$ & 1 & 2 & 2 & - & 5 & 0 & 1 & - & 3.3 \\
\hline AS group $n=20$ & 1 & - & 1 & - & 1 & 14 & - & 1 & 5 \\
\hline PV group $n=77$ & 3 & - & 3 & 1 & 19 & 8 & - & 22 & 28 \\
\hline \multirow[t]{2}{*}{ Total $n=127$} & 4 & 2 & 6 & 1 & 25 & 22 & 1 & 23 & 24 \\
\hline & $3 \%$ & $1 \%$ & $4 \%$ & $0.7 \%$ & $19.6 \%$ & $17.3 \%$ & $0.7 \%$ & $18 \%$ & (18.8)\% \\
\hline
\end{tabular}

$M O$ mechanical obstruction, $A S$ adjunct to surgery group, $P V$ prolonged ventilation group, $A D$ accidental decannulation, $C O$ cannula obstruction, $P T$ pneumothorax, $P M$ pneumomediastinum, $P N$ pneumonia, $D R$ decannulation rate in hospital, $M R$ mortality rate, $R T T$ related to TT, URTT unrelated to TT

Table 2 Complications, decannulation rate and mortality rate at home $(n=81)$

\begin{tabular}{|c|c|c|c|c|c|c|c|c|c|c|c|c|}
\hline & \multirow[t]{2}{*}{$\mathrm{AD}$} & \multirow[t]{2}{*}{$\mathrm{CO}$} & \multirow[t]{2}{*}{$\mathrm{PT}$} & \multirow[t]{2}{*}{ PM } & \multirow[t]{2}{*}{ PN } & \multirow[t]{2}{*}{ T.C.F } & \multirow[t]{2}{*}{ Speech } & \multirow[t]{2}{*}{$\mathrm{DR}$} & \multirow[t]{2}{*}{$\mathrm{LF}$} & \multicolumn{3}{|c|}{ Mortality at home } \\
\hline & & & & & & & & & & RTT & URTT & $\%$ \\
\hline MO group $n=29$ & 1 & 1 & - & - & 1 & 4 & & 17 & 8 & 2 & 2 & $13.7 \%$ \\
\hline AS group $n=5$ & - & - & - & - & - & - & 1 & 0 & 5 & NR & NR & NR \\
\hline PV group $n=47$ & - & 3 & - & - & 12 & - & - & 23 & 20 & NR & 4 & $8.5 \%$ \\
\hline \multirow[t]{2}{*}{ Total $* n=81$} & 1 & 4 & - & - & 13 & 4 & 1 & 40 & 33 & 2 & 6 & 8 \\
\hline & $1.2 \%$ & $5 \%$ & & & $16 \%$ & $5 \%$ & $1.2 \%$ & $49 \%$ & $41 \%$ & $2.4 \%$ & $7 \%$ & $9.8 \%$ \\
\hline
\end{tabular}

$M O$ mechanical obstruction, $A S$ adjunct to surgery group, $P V$ prolonged ventilation group, $A D$ accidental decannulation, $C O$ cannula obstruction, $P T$ pneumothorax, $P M$ pneumomediastinum, $P N$ pneumonia, T.C.F tracheo-cutaneous fistula, $D R$ decannulation rate in hospital, $M R$ mortality rate, $R T T$ related to TT, URTT unrelated to TT, $N R$ not reported, $L F$ lost to follow-up

The largest group was formed by PV having 77 (61\%) patients; their indications are given in Fig. 1. As much as $31(40 \%)$ of the patients were decannulated in this group overall. In this group, the patients were ventilated for 1-48 days with a mean of 24 days before the tracheostomy and 0-37 days with a mean of 9.2 days after the TT. The overall complication rate in this group was $60 \%$, with five patients having more than one complication. All the deaths were unrelated to TT (Tables 1 and 2). 
Fig. 2 Overall outcome of patients with pediatric tracheostomy tube

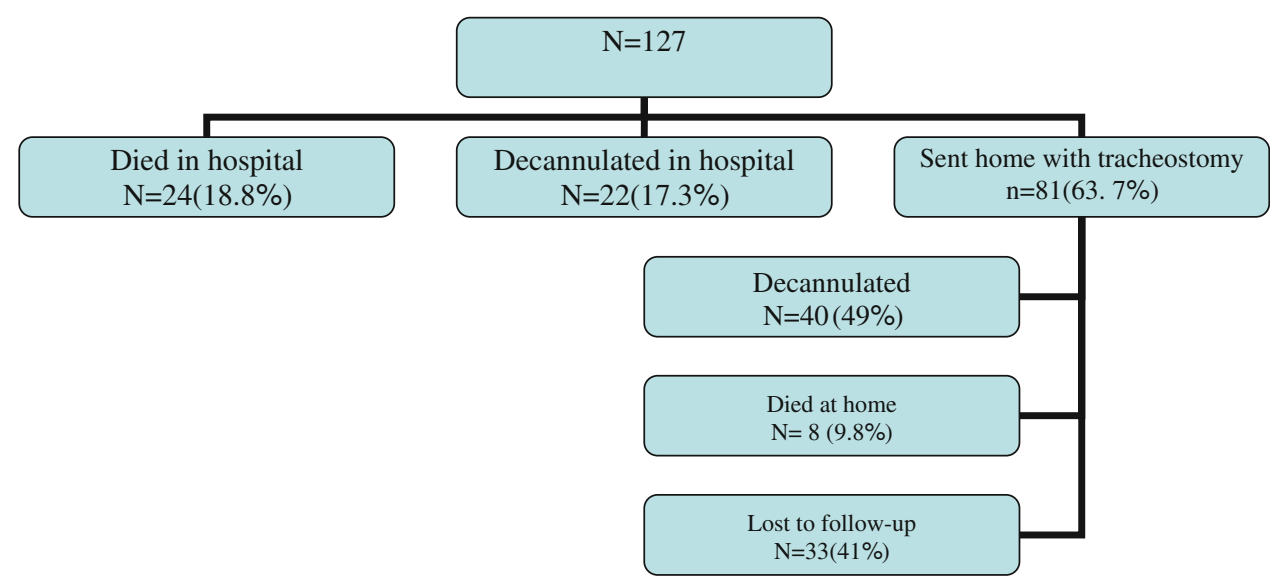

Overall in this study, only 15 patients were ventilated for less than 7 days and the rest for a maximum of 70 days; the range was 1-70 days on ventilation before tracheostomy. Hundred patients $(78.7 \%)$ were successfully weaned off the ventilator; 65 patients $(51 \%)$ could be taken off the ventilator within 7 days of tracheostomy; and 35 (27.5\%) took longer because of their primary lung problems. Fifteen patients $(11.8 \%)$ could not be weaned off the ventilator and succumbed to their chest infections. The mean duration of ICU stay before TT placement was 19 days and after TT was 13 days with a $p$ valve of 0.024 . The days of ventilation before tracheostomy had a mean value of 18 days with a range of 1-70 days. The days of ventilation after tracheostomy had a mean value of 7.7 days and the $p$ value was 0.001 . As much as $22(17.3 \%)$ patients were decannulated in hospital and 81 were sent home with TT. Forty $(49 \%)$ of these patients were successfully decannulated at home (Fig. 2).

The complication rate was $30 \%$ in the hospital and $18.11 \%$ at home. There was only one tracheostomy-related death during the hospital stay of the patient, due to early dislodgement of the tube. Two deaths at home were contributed to TT, taking the overall mortality rate to $2 \%$. There were no peroperative deaths in this study group. Hundred $(78.7 \%)$ patients on the ventilator could be successfully weaned off the mechanical ventilator. The overall decannulation rate for the study was $48.8 \%$.

\section{Discussion}

Pakistan is a developing country where most of the population belongs to the middle or low socioeconomic group. Health insurance is only available to a select group.

There are many studies reported in literature, which look into the indication and complication of pediatric tracheostomy [2-5], but a comprehensive study from any big tertiary care hospital in Pakistan is not available. The indication for pediatric tracheostomy has changed over time from acute upper respiratory tract infection to MO [2], the trend which was not replicated in our study. The main indication of tracheostomy in our study population was PV of $61 \%$, which was dissimilar to that reported in literature [2].The need for early TT in these patients arises from the need to save cost, as financial assistance is available to only a select few of such patients. Prolonged hospitalization has a very major impact on the economic burden of the families, as well as the country. The second reason is to prevent nosocomial infections. The PV group had higher morbidity, both in the hospital as well as at home. The reasons could have been that their tracheostomies were kept for a longer time as compared to other groups. The mean duration of ICU stay before TT placement was 19 days and after TT 13 days, with a $p$ value of 0.024 . The days of ventilation before tracheostomy had a mean value of 18 days with a range of 1-70 days. The days of ventilation after tracheostomy had a mean value of 7.7 days. The $p$ value was 0.001 , which signifies positive benefit of tracheostomy in taking patients off ventilator.

Michele et al. had reported an overall morbidity rate of $77 \%$ in his study of 142 patients [11]. Our overall morbidity rate related to TT in hospital and at home was 59\%. Messineo et al. [8] in his study on 34 tracheostomies mentioned upper respiratory tract infection as the major complication and reported no death. In our study, the overall complication rate in the hospital was $30.7 \%$ and at home $18.11 \%$, which was more in the PV group. This rate is lower than that reported in literature. The lower rate of complications in the home care part is probably related to the lower rate of reporting of such incidents. John et al. have mentioned a complication rate of $21.5 \%$ in their study of 149 tracheostomies. There were no reported deaths [14]. Dutton et al. mentioned a mortality of $0-3.4 \%$ [12]. The overall mortality rate related to tracheostomy was $2 \%$ in our study, which is comparable to reports in literature. 
Home care has major contribution toward subsequent decannulation and reducing the tracheostomy-related morbidity [9]. Therefore, we need to set up our own practice guidelines for home-based TT care with regard to the economic, social and educational background of the parents and to practice them according to our own environment. To establish these guidelines, a close follow-up system for these patients is a prerequisite for its success and safety. Better training programs for parents could then be evolved, which would provide not only good home tracheostomy care, but also educate them to administer cardiopulmonary resuscitation if needed and prepare them in case of emergency. Home care of tracheostomy has been found to be safe [4]. Okamoto et al. [10] reported a mortality of two deaths per 100 months of home tracheostomy. Recent literature has also reported zero mortality for tracheostomies managed at home $[11,12]$. We do not have the luxury of having a home care nurse come to our help, unlike this facility being available in most developed countries. In a study of 44 patients by Messineo et al. [8], home care was arranged by the hospital in $77 \%$ of cases. In this study population, there were no tracheostomy-related deaths; home nursing helped significantly to decrease the stress of the parents caring for their tracheostomy-dependent children at home [13].

Our study has a high rate of loss to follow-up (41\%). Our hospital being a large tertiary care referral center in the region gets patients not only from all parts of Pakistan, but also from neighboring countries such as Afghanistan, Iran and the United Arab Emirates. We have very good inpatient medical records system; but when the patient is discharged, our present systems of communications are inefficient to trace them back if they do not present for subsequent treatment. Another reason could be that our country does not have a developed system of referral and feedback to the primary physician, which is now a wellestablished practice in the developed countries. A better system needs to be developed to decrease the number of patients lost to follow-up.

\section{Conclusion}

Tracheostomy is a useful and life-saving procedure in the pediatric population. It helps to reduce the duration of endotracheal intubation and ventilation and earlier discharge of patients from the ICU setting. In our study, the $p$ value was 0.001 and 0.024 , respectively. The mortality rate was $2 \%$ in our population. Home care is a viable option in children requiring tracheostomy for prolonged periods even in our setting where home nursing help is difficult to arrange. It requires proper training of motivated parents to decrease complications related to TT.

\section{References}

1. McMurray JS, Prescott CAJ (1996) Tracheostomy in the pediatric patient. In: Cotton RT, Myer CM (eds) Practical pediatric otolaryngology. W.B. Saunders Company, Philadelphia, pp 575-592

2. Trimezey MA (1992) Indications for pediatric tracheostomy in Faisalabad. Pak J Otolaryngol 8:16-18

3. Kremer B, Botos-Kremer AI, Eckel HE, Schlondorff G (2002) Indications, complications and surgical techniques for pediatric tracheostomies-an update. J Pediatr Surg 37(11):1556-1562

4. Mansoor T, Rashid D, Haq AU (2000) Complications of tracheostomy. Pak Armed Forces Med J 50(1):17-19

5. Markstrom A, Sundell K, Lysdahl M, Andersson G, Schedin U, Klang B (2002) Quality of life evaluation of patients with neuromuscular and skeletal diseases treated with noninvasive and invasive home mechanical ventilation. Chest 122(5):1695-1700

6. Duncan BD, Howell LJ, Delorimier AA, Adzick NS, Harrison MR (1992) Tracheostomy in children with emphasis on home care. J Pediatr Surg 27(4):432-435

7. Sherman LP, Rosen CD (1990) Development of a preschool program for tracheostomy-dependent children. Pediatr Nurs 16(4):357-361

8. Messineo A, Giusti F, Narne S, Mognato G, Antoniello L, Guglielmi M (1995) The safety of home tracheostomy care for children. J Pediatr Surg 30(8):1246-1248

9. Gray RF, Todd W, Jacobs IN (1998) Tracheostomy decannulation in children: approaches and techniques. Laryngoscope 108:8-12

10. Okamoto E, Fee WE Jr, Boles R, Calcaterra TC, Dobie RA, Steadman MG (1977) Safety of hospital vs home care of infant tracheotomies. Trans Am Acad Ophthalmol Otolaryngol 84(1): 92-99

11. Carr MM, Poje CP, Kingston L, Kielma D, Heard C (2001) Complications in pediatric tracheostomy. Laryngoscope 111: 1925-1928

12. Dutton JM, Palmer PM, McCulloch TM, Smith RJ (1995) Mortality in pediatric patient with tracheostomy. Head Neck 17:403-408

13. Tom LWC, Miller L, Wetmore RF, Handler SD, Potsic WP (1993) Endoscopic assessment in children with tracheostomies. Arch Otolaryngol Head Neck Surg 119:321-324

14. Park JY, Suskind DL, Prater D, Muntz HR, Lusk RP (1999) Maturation of the pediatric tracheostomy stoma: effect on complications. Ann Otol Rhinol Laryngol 108:1115-1119 